\title{
Fluoroscopic Imaging Guides of the Posterior Pelvis Pertaining to lliosacral Screw Placement
}

\author{
Bruce H. Ziran, MD, Ajay D. Wasan, MD, David M. Marks, MD, Steven A. Olson, MD, and \\ Michael W. Chapman, MD
}

Background: This study correlates anatomic landmarks with radiographic densities and radiolucencies of the posterior pelvis, which assists in percutaneous iliosacral screw placement.

Methods: Radio-opaque markers were placed on selected surfaces of cadaveric specimens and rotated under a fluoroscopic beam. Identified surfaces were described with angular measurements and anatomic characteristics.

Results: The anterior border of the $\mathrm{S1}$ body is not parallel to the anterior border of the S1 ala. The anterior surface of the S1 ala is found on the inlet view $\left(\sim 49^{\circ}\right)$ by the superimposition of the S1 and S2 alar ridges. Superior surface of the $\mathrm{S} 1$ ala is difficult to visualize fluoroscopically because of the highly oblique outlet angle required by fluoroscopy $\left(\sim \mathbf{8 0 ^ { \circ }}\right)$. The superior ridge of the $\mathrm{S1}$ foramen can best be seen during an outlet view when the projection of the superior pubic symphysis is superimposed on the S2 foraminal image. Posterior bony surfaces of the ilium, foraminal region of sacrum, and sacral body are difficult to identify, as are the bony surfaces of the sacral foramina. During screw placement, the narrowest area of bone occurs in the sacral foraminal region.

Conclusions: Use of predetermined angles for inlet and outlet views may not provide optimal visualization of relevant bony landmarks during iliosacral screw insertion. Because of variable sacral morphologies and lumbosacral lordosis, we propose using inherent landmarks to help visualize important landmarks during screw placement. Altering the fluoroscopic angle to superimpose the anterior S1 and S2 alar opacities allows the best visualization of the anterior boundary of the S1 ala. The superior bony surface of the S1 foramen is best visualized on the outlet view.

Key Words: Fluoroscopic images, Posterior pelvis, Iliosacral screws, Osseous landmarks.

J Trauma. 2007;62:347-356. liosacral screw fixation has become a popular technique for treating unstable injuries of the pelvis that involve the posterior ring. ${ }^{1-7}$ In this procedure, one or two large screws (6.5-7.3 $\mathrm{mm}$ diameter) are inserted under fluoroscopic guidance through the ilium, across the sacroiliac articulation, and into the superior sacral vertebral bodies using percutaneous or open techniques. There is limited published information on the fluoroscopic guides used for iliosacral screw placement. Previous literature recommends use of standard lateral, anterior-posterior, and inlet/outlet (approximately 40-50 degrees from the anterior-posterior direction) images, or by using superimposition of sacral bodies or en-face viewing of neural foramina. ${ }^{8-12}$ However, these recommendations do not account for variances in sacral morphology, lumbosacral lordosis, or soft-tissue obscuration. Furthermore, there are no studies that attempt to correlate the osseous landmarks with their corresponding fluoroscopic images. Addition-

Submitted for publication September 23, 2005.

Accepted for publication March 24, 2006.

Copyright () 2007 by Lippincott Williams \& Wilkins, Inc.

From the Department of Orthopaedic Surgery, St. Elizabeth Health Center (B.H.Z.), Youngstown, OH; the Department of Orthopaedic Surgery, University of California, Davis (A.D.W., S.A.O., M.W.C.), Sacramento, CA; and the Duke University Medical Center (D.M.M.), Durham, NC.

Address for reprints: Bruce H. Ziran, MD, Director of Trauma, Northeast Ohio Universities College of Medicine, St. Elizabeth Health Center, 1044 Belmont Avenue, Youngstown, OH 44501-1790; email: bruce_ziran@ hmis.org.

DOI: 10.1097/01.ta.0000229789.18211.85 ally, poor fluoroscopic image quality coupled with the potential misinterpretation of the osseous anatomy on the fluoroscopic monitor can result in complications such as malpositioned screws or injury to adjacent neurovascular structures. $^{5,13-15}$

Although the osteology of the sacrum has been characterized, we have not found a study that correlated the anatomic landmarks of the posterior pelvis to their corresponding fluoroscopic images. ${ }^{16,17}$ Because of individual variation in posterior pelvis morphology and alignment, proper fluoroscopic visualization of pertinent structures requires correlation of radiographic findings to osseous anatomy, and will require adjusting the angle of the X-ray beam to optimally view each patient's pertinent anatomy. The boundaries of the sacral foraminal region during screw placement are the superior and anterior S1 ala, the superior bony surface of the S1 foramen, and the anterior spinal canal. Safe screw placement is usually dependent on accurately identifying the critical boundaries of this volume of bone fluoroscopically. Although there are studies that characterize the computed tomography of this volume of bone used for iliosacral screw placement, ${ }^{18-21}$ there is reference only to the iliac cortical density or alar slope on the lateral view when utilizing intraoperative fluoroscopy. ${ }^{7}$ The purpose of the present study is to associate anatomic landmarks with their corresponding fluoroscopic images and to provide a guide for accurately visualizing specific important landmarks pertinent to the placement of iliosacral screws into the S1 body. 


\section{MATERIALS AND METHODS}

We studied 17 whole cadaveric adult pelves (5 female, 12 male) obtained from a human skeletal collection. The specimens had no evidence of neoplasm, trauma, degenerative disease, or dysplasia. The L5 vertebra had been removed from them, but the sacroiliac ligaments were preserved. Detailed premorbid specimen data were not available for review. Specimens with alar dysmorphisms such as lumbarization or sacralization of the lumbosacral elements were excluded.

\section{Marker Fabrication and Placement}

The following structures were considered relevant to iliosacral screw fixation: the superior and anterior $\mathrm{S} 1$ ala, the upper border of the $\mathrm{S} 1$ foramen, the anterior $\mathrm{S} 1$ body, the superior S1 endplate, the posterior S1 body, and the sacroiliac joint. Portions of these structures were identified with either a metal pointer or radio-opaque markers in a fashion analogous to the studies of Letournel, who identified the irregular surfaces of the periacetabular anatomy. ${ }^{22}$ The S2 and S3 bodies were not characterized because of the infrequency and danger of fluoroscopic screw placement into those areas.

Because many of these structures are curved surfaces with some planar characteristics, they will produce radiodense lines only when viewed tangentially during fluoroscopic imaging (OEC model 7600, Salt Lake City, UT). To identify the tangential view of such surfaces (and thus identify the angular orientation of the fluoroscopic beam), pairs of parallel malleable radio-opaque markers were attached to the surfaces. With this technique, the clinical scenario of using tangential fluoroscopic imaging to detect violation of such a surface by a malpositioned screw is approximated. The markers were made of 2-mm lead wire that was cut precisely to 2 $\mathrm{cm}$ and secured between two $2-\mathrm{cm}$ square sections of foam tape. In doing so, they remain parallel but can be slightly molded to contour to nonplanar surfaces. The markers were contoured to be flush with the bony surface of interest and were held on the bone with another piece of foam tape (Fig. 1). When these wires were seen en face (perpendicular to the plane created by the two wires), they appeared as two parallel lines, but as the radiographic beam became parallel to the plane of the markers, the fluoroscopic image of these markers changed to a single line, indicating tangentiality. Measurements and appropriate observations were recorded as described below.

When an irregular surface resulted in some deformity of the marker pair, only the portion of the markers directly over the central aspect of the surface being imaged was evaluated. For example, on the anterior sacral ala, there is a concave surface from medial to lateral and a convex surface (transition of superior to anterior ala) from cephalad to caudad. Likewise, for the superior portions, there can be a convexity or concavity depending on sacral morphology. In addition, the peripheral aspects of the marker pairs are not coplanar with the central portions. Because of such variations in the contour of various surfaces, we placed the markers in the central-most

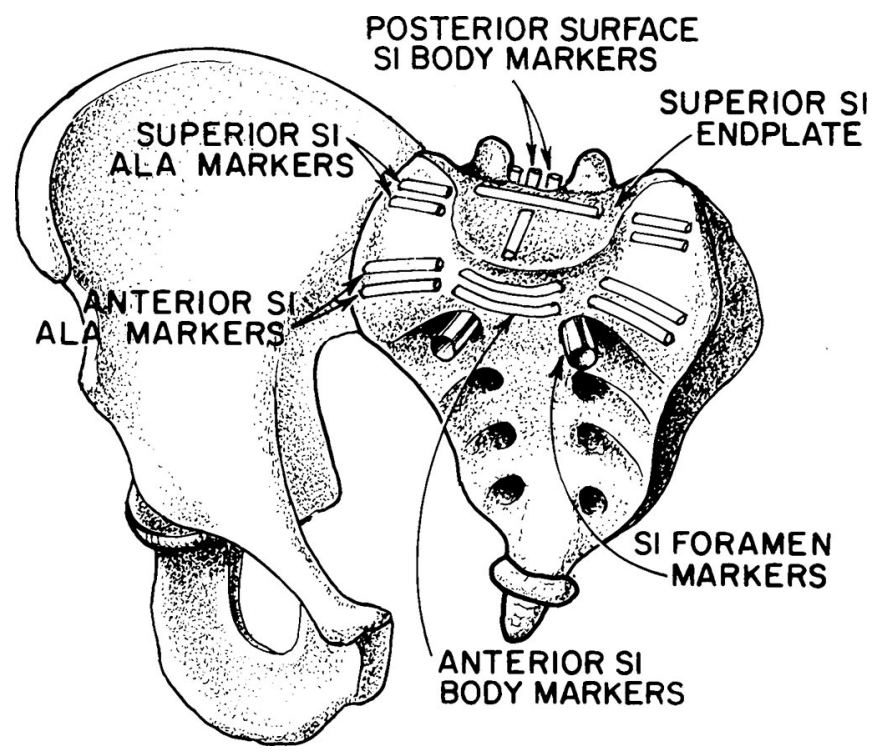

Fig. 1. Illustration of the placement of marker pairs used to define a plane. When the fluoroscopic beam becomes coplanar to each pair, they become superimposed and appear as a single line.

portion of the area of interest to best identify the landmark of interest. Markers were consistently centered over areas of interest, and we thought that this technique was reproducible and satisfactorily accurate. When necessary we repeated marker placement and measurements to verify accuracy. Figures 2 to 4 are fluoroscopic images and schematic illustrations of markers and structures.

Additionally, a metal pointer was used to more precisely identify certain osseous and fluoroscopic landmarks. The metal pointer was moved to touch or enter a particular area during imaging. In this fashion, correlations between an osseous landmark and fluoroscopic image were localized. Various radiodensities usually seen with fluoroscopic imaging were identified in this fashion. For the foramen, thin metal sheets that were $2 \mathrm{~cm}$ long and wide were rolled up into a cylinder. They were placed into each foramen and the resilience of the metal caused the tubes to open to match the diameter of the foramen. This not only helped secure the tubes in the foramen but also helped to establish their directional axis.

\section{References for Measurements}

Most measurements were made in the sagittal plane and referenced to the perpendicular to the frontal plane (anteroposterior [AP] image axis). The frontal plane was defined as the plane created by three landmarks: the two anterior-superior iliac spines and the pubic symphysis (Fig. 5). This reference plane has been previously described and proposed in anatomic texts. ${ }^{23}$ Sagittal measurements with the beam source cephalad to this axis were considered the "inlet" direction, and those with the beam source caudad to this direction were considered the "outlet" direction. 


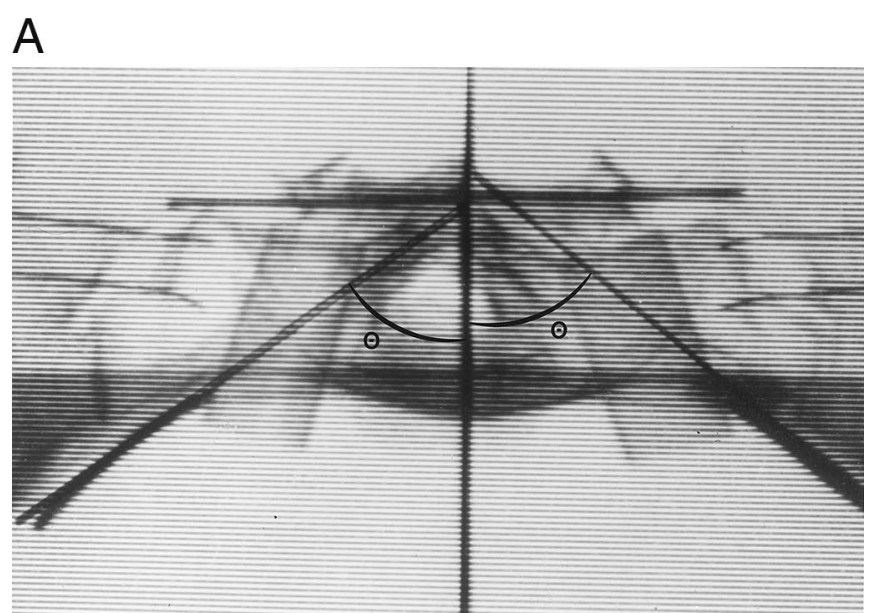

B

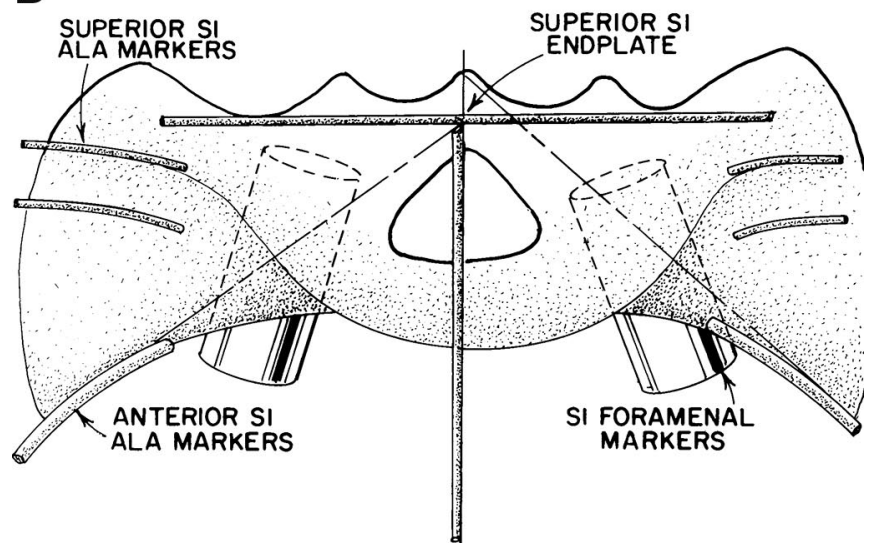

Fig. 2. (A) Inlet image from monitor showing the angular divergence of the anterior S1 ala from the midline, occurring when the anterior alar markers are superimposed and approximate a straight line. (B) Schematic illustration of the image shown in A, clarifying structures and details.

Two structures, the superior S1 ala and the superior aspect of S1 foramen, had oblique components to their orientation and were therefore described relative to the sacrum. These structures were referenced off the superior $\mathrm{S} 1$ endplate (Fig. 6). Using the superior S1 endplate as a reference, a sacral reference was devised that allowed such measurements to be described easier. For example, there is a "frontal" plane of the sacrum as well as the "sagittal". Although not the anatomic frontal plane described previously, the measurements are easier to understand in relation to the sacrum than to the pelvis. This description was used for landmarks such as the superior cortex of the $\mathrm{S} 1$ ala and sacroiliac joint orientation near the area also known as the iliac cortical density on the lateral view.

\section{Fluoroscopic Evaluation}

Each pelvis was securely mounted in a radiolucent Plexiglas mounting apparatus that allowed uniaxial rotation about a transverse axis of the pelvis. The pelves were secured with
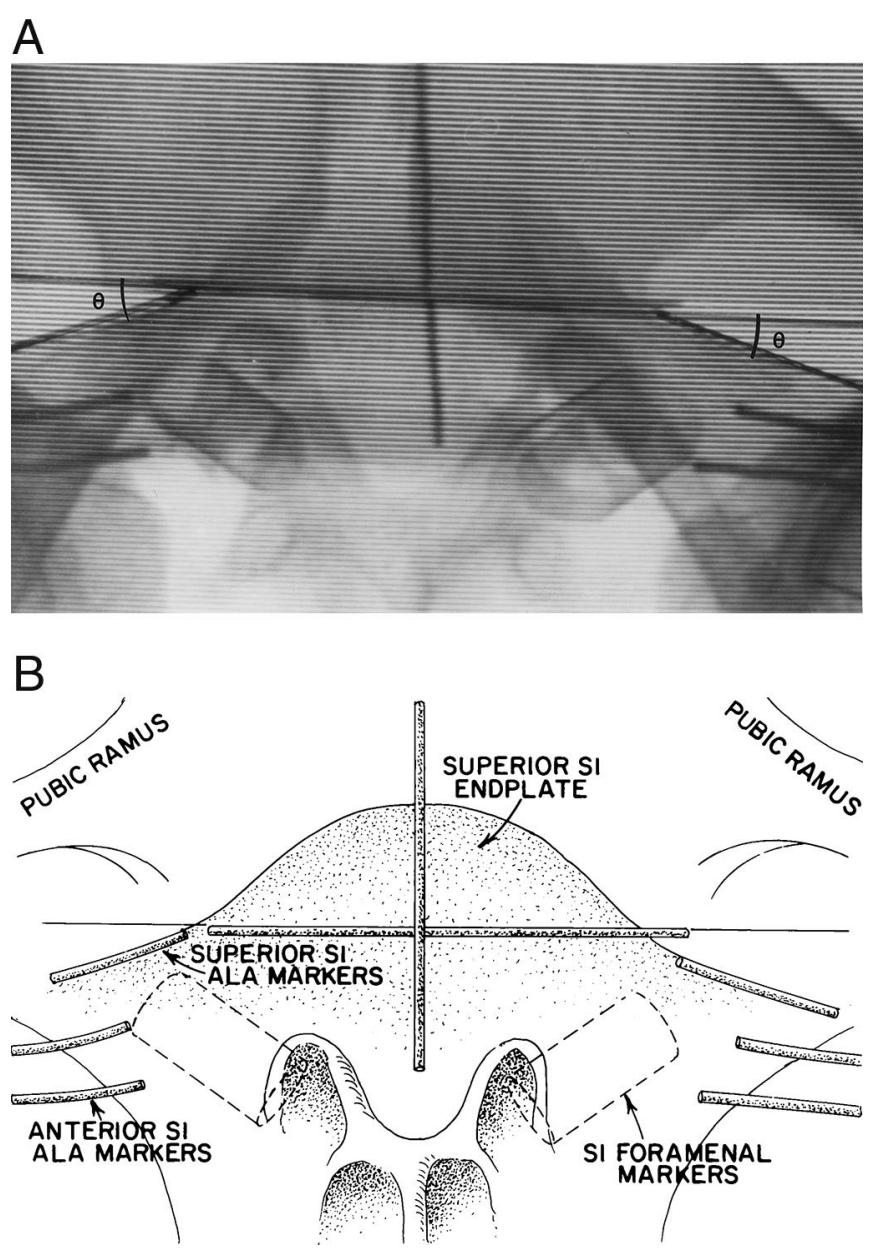

Fig. 3. (A) Outlet image from monitor showing the slope of the superior sacral ala in the frontal plane resulting when superior alar markers are superimposed and approximately a straight line. (B) Schematic illustration of the image shown in A, clarifying structures and details.

external fixation pins placed into the iliac wings and posterior superior spines (Fig. 7). A radiolucent piece of Plexiglas was secured to make contact with the anterior superior iliac spines and pubic tubercles (parallel to the frontal plane). The pelves were adjusted in the frame so that the axis of rotation was at the level of the sciatic notch for each specimen. Angular measurements were made with a metal goniometer with 1-degree increments securely mounted to the apparatus. Each pelvis was calibrated so that a true anterior-posterior image registered as 0 degrees (in inlet or outlet angulation). When the pelves were rotated in the frame during fluoroscopy, the goniometer would measure the corresponding inlet or outlet angle.

During testing, the specimen was rotated in the apparatus under live fluoroscopy with markers attached to different locations. When the marker pairs became superimposed to appear as a single line, the angular value of the pelvis in the frame for that respective osseous structure was measured with the goniometer mounted to the apparatus. The superimposition of the markers represents the tangency of the fluoroscopic beam 
A

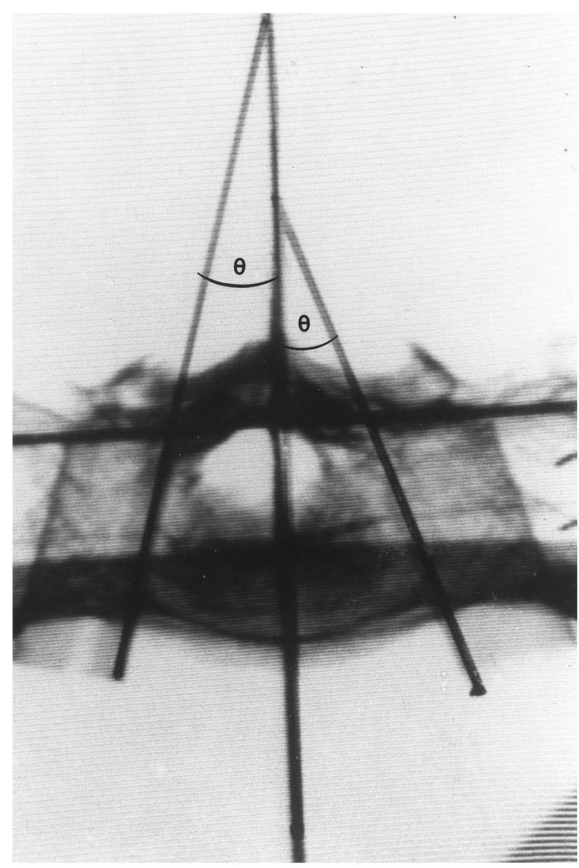

B
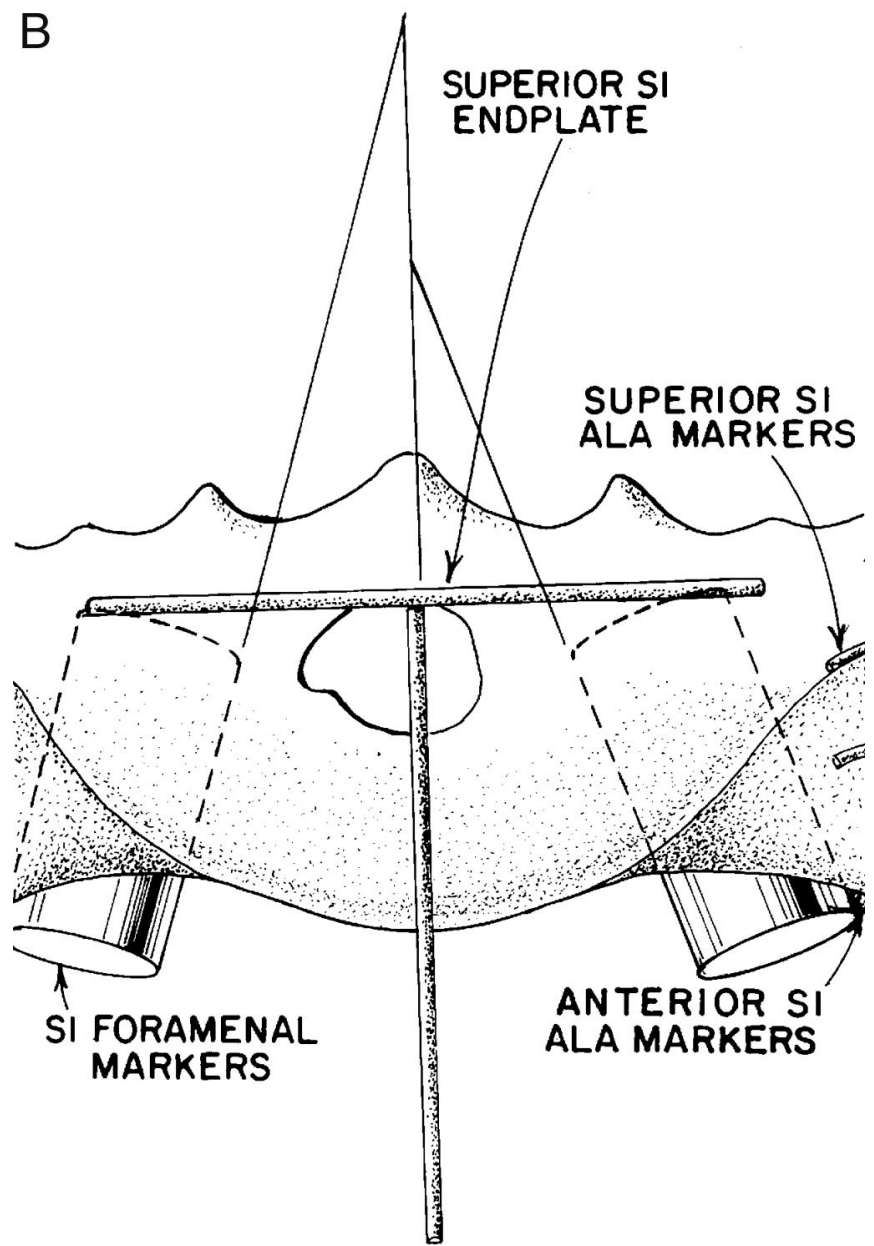

Fig. 4. (A) Angular divergence of the S1 foramen from the midline. (B) Schematic illustration of image shown in A, clarifying structures and details.

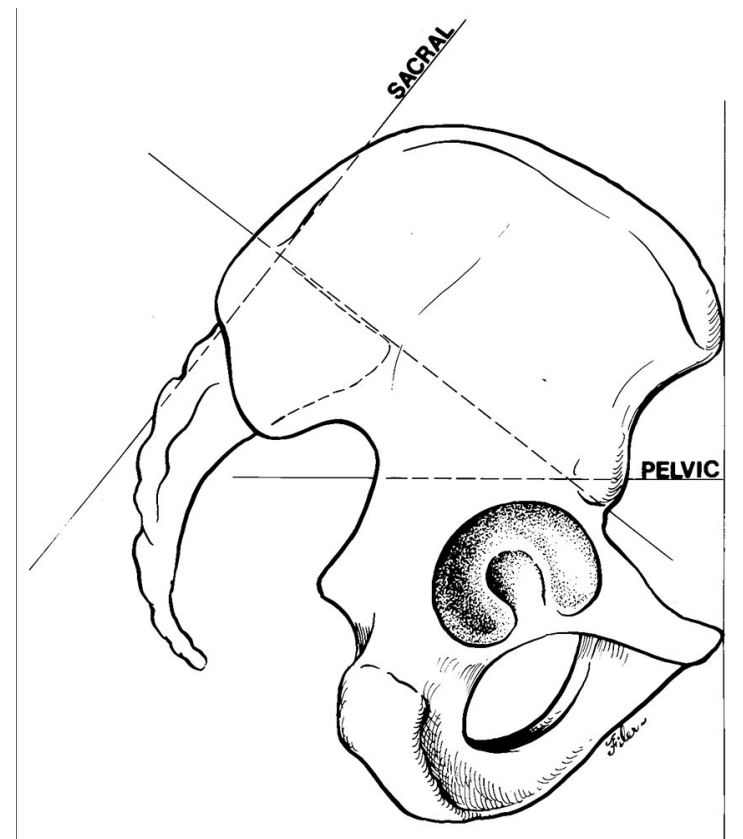

Fig. 5. The pelvic system: the anterior-to-posterior direction is orthogonal to the plane created by the anterior superior iliac spines and the pubic symphysis.

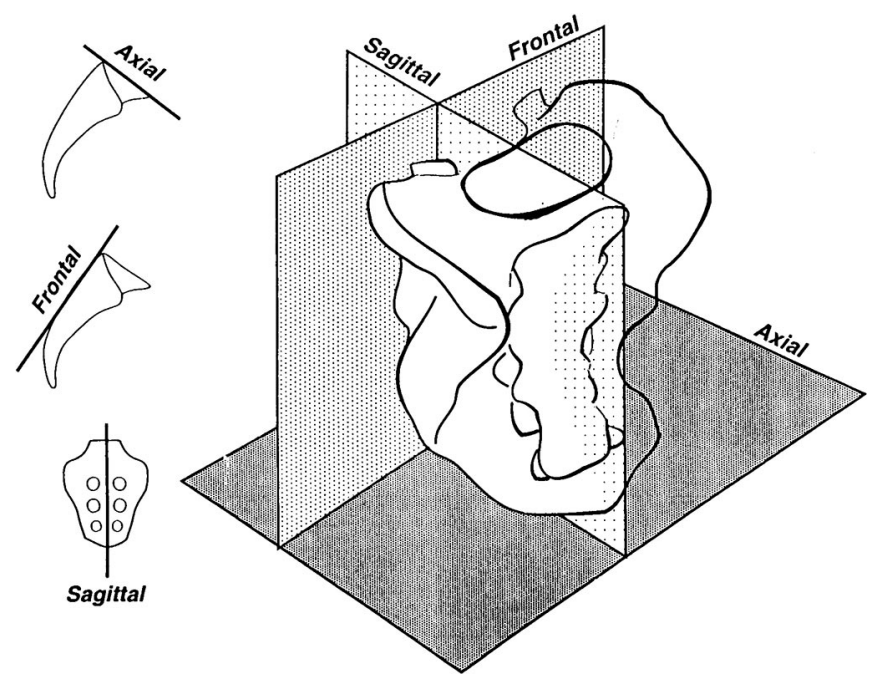

Fig. 6. The sacral system is based on the superior endplate of the first sacral vertebra. The transverse plane is parallel to the first sacral endplate, and both other planes are orthogonal to the axial.

with the osseous surface. This technique was used for all structures that could be measured with sagittal plane rotations of the pelvis and was the main focus of the study.

Other structures with oblique orientations that could not be easily identified in the pelvic reference system were measured using the sacral reference system as described previously. Measurements that were clinically relevant were generally those measurable in the sagittal and transverse planes and were reported below. During the part of the study where a pointer was used, the following fluoroscopic lines 


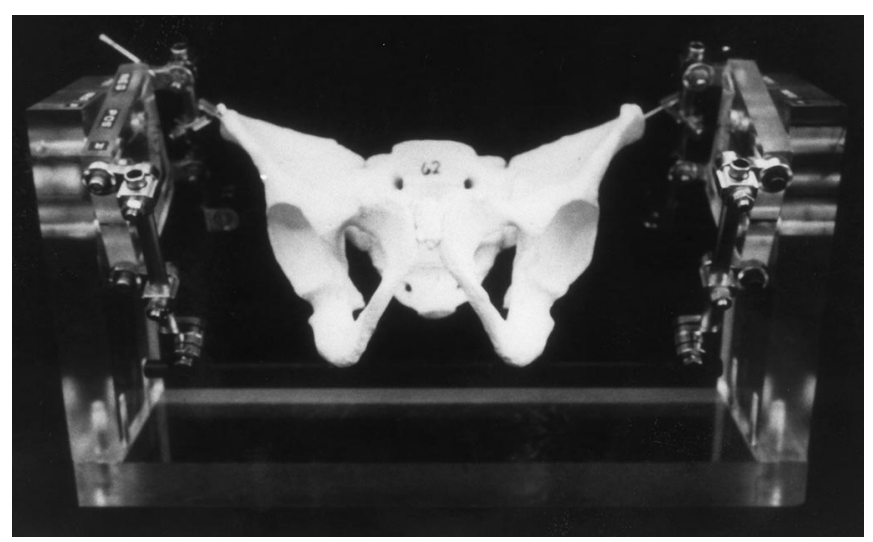

Fig. 7. A pelvic specimen, mounted in an apparatus that allows 360-degree rotation, is stabilized with Schanz pins.

were localized on the sacrum: the radiolucent and radiodense lines around the anterior and posterior of the S1 and S2 foramina on the anteroposterior view; the radiodense lines located centrally (thought to represent the anterior cortices of the sacral vertebral bodies) and laterally (thought to represent the anterior cortex of the sacral ala) on the inlet view; and the radiodense line, described as the "alar slope" or "iliac cortical density" on the lateral view. By moving the pointer, we could place it directly on a fluoroscopic density or radiolucency and determine the superficial osseous location that best matched the radiographic landmark.

With all measurements, three observers separately measured each structure and landmark, and their three measurements then were averaged for each item; side to side differences were also compared. Statistical analysis of side to side differences was done with a two-tailed $t$ test with $p<0.05$ as the level of significance.

\section{RESULTS}

In the inlet direction, the markers on the anterior surface of the $\mathrm{S} 1$ body became tangent to the fluoroscopic beam at smaller inlet angles than the markers on the anterior S1 ala and posterior S1 body. The latter two structures were nearly parallel in all specimens. The pubic rami became superimposed upon each other between 30 and 40 degrees on the inlet view.

In the outlet direction, markers on the superior S1 endplate became coplanar at smaller outlet angles than the markers on the superior cortex of the S1 ala. The sciatic notch was located at the level of the S2 foramen on all outlet views but was obscured by superimposed structures with outlet view of greater than 30 degrees. The posterior spinal foramen was directed posteriorly and cephalad and divergent from the midline, but the thin sacral lamina prevented use of small metallic tubes to accurately characterize their true orientation. The sacroiliac joint space could be best visualized when the radiographic beam was directed obliquely 10 to 20 degrees in the transverse plane (direction for obturator oblique views).

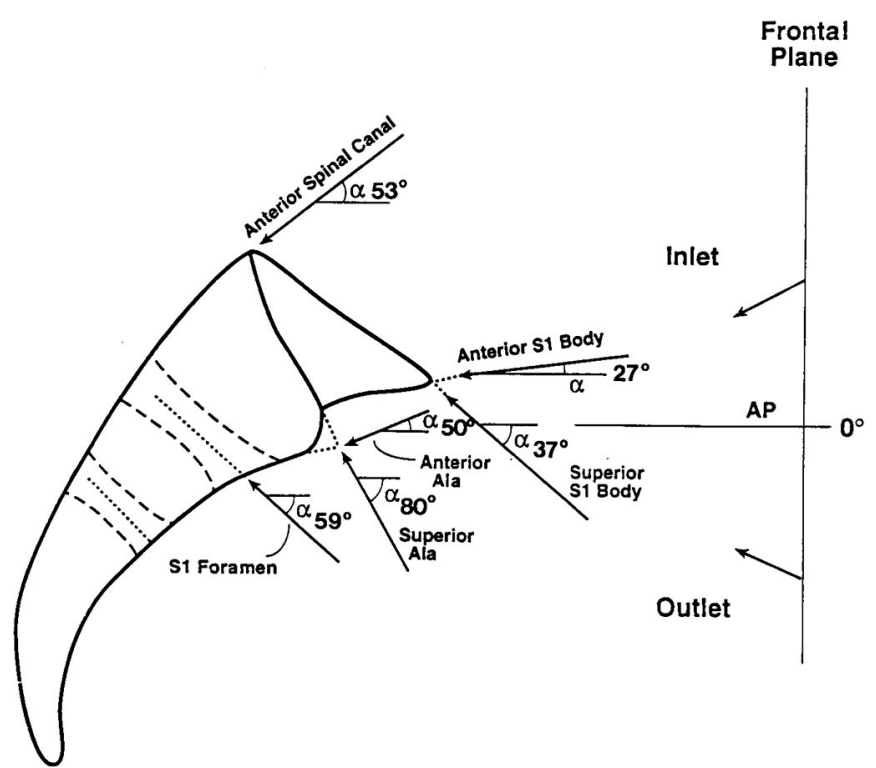

Fig. 8. Schematic lateral view of the sacrum depicting the measured osseous surfaces and their orientations to the frontal plane of the pelvis.

\section{Angular Measurements}

The angular orientations of the anterior S1 body surface, anterior cortex of the S1 ala, and posterior S1 surface were oriented $27 \pm 11$ degrees, $50 \pm 8$ degrees, and $53 \pm 6$ degrees, respectively, inlet from the anterior-posterior direction. The orientation of the superior cortex of the S1 ala in the pelvic sagittal plane was approximately $80 \pm 7$ degrees, outlet from the anterior-posterior direction. The plane of the superior endplate of $\mathrm{S} 1$ and the axes of the $\mathrm{S} 1$ foramen were oriented $37 \pm 6$ and $59 \pm 8$ degrees, respectively, outlet from the anterior-posterior direction (Fig. 8). The planes of the posterior S1 body and superior S1 endplate were nearly perpendicular, and those of the anterior cortex of the S1 ala and posterior S1 body (representing the anterior border of the spinal canal) were nearly parallel.

In the sacrum, the frontal plane inclination of the superior cortex of the $\mathrm{S} 1$ ala was approximately $9 \pm 10$ degrees but varied between a 12-degree superior and a 22-degree inferior inclination. Concave alar morphologies were associated with inferior and convex alar morphologies with superior inclinations, respectively.

Table 1 summarizes the angular measurement data. No side-to-side differences were noted in any of the measured landmarks. The only male-to-female difference noted was the S1 foraminal angle in the sagittal plane, which was oriented more in the anterior-posterior direction in female pelves (left side, $p<0.05$; right side, $p<0.01$ ). The angular values of most osseous landmarks had inter-specimen variances between $7 \%$ and $25 \%$. The osseous planes exhibiting the least variance were sagittal plane measurements of the superior cortex of the S1 ala (coefficient of variance, $8 \%$ to $9 \%$ ) and anterior S1 ala (10\%). The osseous planes that had the great- 


\begin{tabular}{|c|c|c|c|}
\hline Structure (plane measured in, view) & Male & Female & Total \\
\hline Anterior SI body (sagittal, inlet) & $29(12)$ & $24(12)$ & $27(12)$ \\
\hline $\begin{array}{l}\text { Anterior spinal canal (sagittal, } \\
\text { inlet) }\end{array}$ & $54(5)$ & $50(7)$ & $53(5)$ \\
\hline Superior SI body (sagittal, outlet) & $36(5)$ & $42(5)$ & $37(5)$ \\
\hline Anterior ala (left sagittal, inlet) & $50(17)$ & $47(19)$ & $49(17)$ \\
\hline Anterior ala (right sagittal, inlet) & $49(14)$ & $52(17)$ & $50(15)$ \\
\hline $\begin{array}{l}\text { Anterior ala (left axial from } \\
\text { midline, inlet) }\end{array}$ & $57(7)$ & $53(5)$ & $56(7)$ \\
\hline $\begin{array}{l}\text { Anterior ala (right axial from } \\
\text { midline, inlet) }\end{array}$ & $54(6)$ & $62(7)$ & $54(5)$ \\
\hline $\begin{array}{l}\text { Superior SI joint (left axial from } \\
\text { midline, inlet, photo) }\end{array}$ & $11(5)$ & $10(5)$ & $11(6)$ \\
\hline $\begin{array}{l}\text { Superior SI Joint (right axial from } \\
\text { midline, inlet, photo) }\end{array}$ & $11(4)$ & $12(6)$ & $11(5)$ \\
\hline $\begin{array}{l}\text { Anterio SI joint (left coronal from } \\
\text { midline, outlet, photo) }\end{array}$ & $15(2)$ & $16(6)$ & $15(3)$ \\
\hline $\begin{array}{l}\text { Anterio SI joint (right coronal } \\
\text { from midline, outlet, photo) }\end{array}$ & $16(3)$ & $15(6)$ & $15(4)$ \\
\hline $\begin{array}{l}\text { S1 foraminal axis (left sagittal, } \\
\text { outlet) }\end{array}$ & $57(9)$ & $63(5)$ & $59(5)$ \\
\hline $\begin{array}{l}\text { S1 foraminal axis (right sagittal, } \\
\text { outlet) }\end{array}$ & $57(9)$ & $61(6)$ & $58(9)$ \\
\hline $\begin{array}{l}\text { S1 foraminal axis (left axial from } \\
\text { midline, inlet) }\end{array}$ & $23(4)^{\star}$ & $17(4)^{\star}$ & $22(5)$ \\
\hline $\begin{array}{l}\text { S1 foraminal Axis (right axial } \\
\text { from midline, inlet) }\end{array}$ & $23(5)^{\dagger}$ & $16(2)^{\dagger}$ & $21(5)$ \\
\hline Superior ala (left sagittal, outlet) & $81(7)$ & $78(8)$ & $80(7)$ \\
\hline Superior ala (right sagittal, outlet) & $80(7)$ & $78(9)$ & $79(7)$ \\
\hline Superior ala (left coronal, outlet) & $10(11)$ & $6(15)$ & $5(12)$ \\
\hline Superior ala (right coronal, outlet) & $10(9)$ & $11(13)$ & $11(10)$ \\
\hline
\end{tabular}

Data are degrees (SD).

${ }^{*} p<0.05$.

${ }^{+} p<0.01$.

est discrepancies were the orientations of the anterior surface of the S1 vertebral body in the sagittal plane (coefficient of variance, $43 \%$ ), the orientation of the superior portion of the sacroiliac joint in the sacral axial plane (47\% to 52\%), and the orientation of the superior S1 ala in the sacral frontal plane (97\% to $140 \%)$.

\section{Landmark Identification \\ Inlet Views}

A partial inlet view was used to demonstrate the crescent-shaped radiodense lines in the foraminal regions that represent the superior cortical bone of the opening (or cephalad ridge) of the anterior foramina. These lines were well visualized with inlet views and, as described previously, defined the plane of the anterior S1 ala when superimposed. In the central area, the radiodense lines on the inlet views corresponded to cortical rims of the sacral body interspaces. The sacral promontory, because of its thin wedge shape, is not as radiopaque as denser structures and this could not be well visualized fluoroscopically. The posterior S1 body was poorly seen on all views. Given the kyphotic course of the sacral canal, this part of the sacrum is difficult to differentiate from more caudad portions.
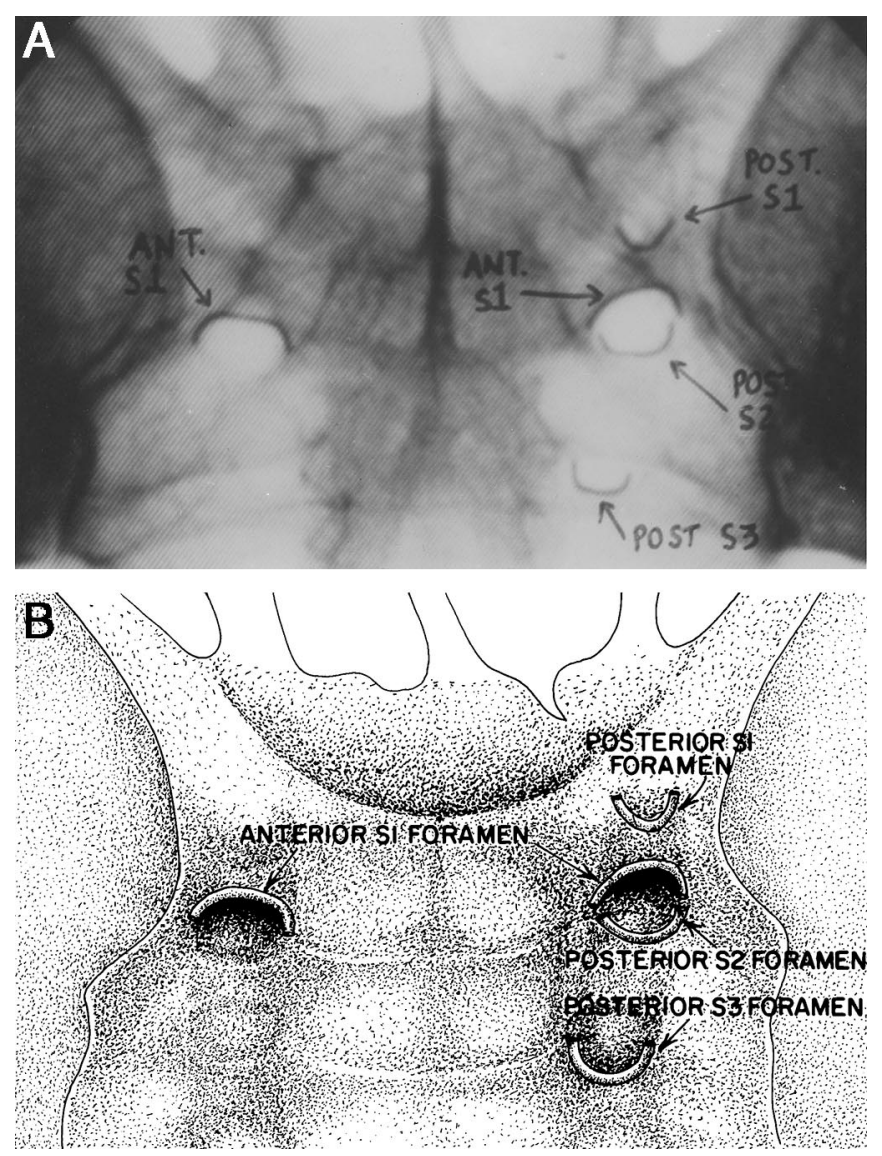

Fig. 9. (A) Anterior-posterior image with hemispheric markers outlining sacral foraminal ridges. (B) Schematic illustration of the image shown in A, clarifying the structures and details.

\section{Anterior-Posterior Views}

On the anterior-posterior view, the radiodense area caudad to the $\mathrm{S} 1$ foraminal crescent, which appears on radiograph to be the inferior border of the $\mathrm{S} 1$ foramen, is in actuality the inferior border of the posterior $\mathrm{S} 2$ foraminal outlet (Fig. 9A, B).

\section{Outlet Views}

The superior S1 alar cortex, which represents the superior border of the volume used for screw placement, needed a very large outlet angle ( $\sim 80$ degrees) to be viewed tangentially. The superior portion of the S1 foramen (representing the inferior border of the same volume) became best visualized as the fluoroscopic image of the superior pubic symphysis approached superimposition of the inferior aspect of the $\mathrm{S} 1$ foramen (approximately over the S2 foramen).

\section{Lateral View}

On the lateral view, the radiodense line (normally termed the lateral sacral alar slope or iliac cortical density) was in actuality the tangential projection of cortical bone around the sacroiliac joint. As the pointer was moved from the inner iliac cortex across the sacroiliac joint and onto the ala, we could 

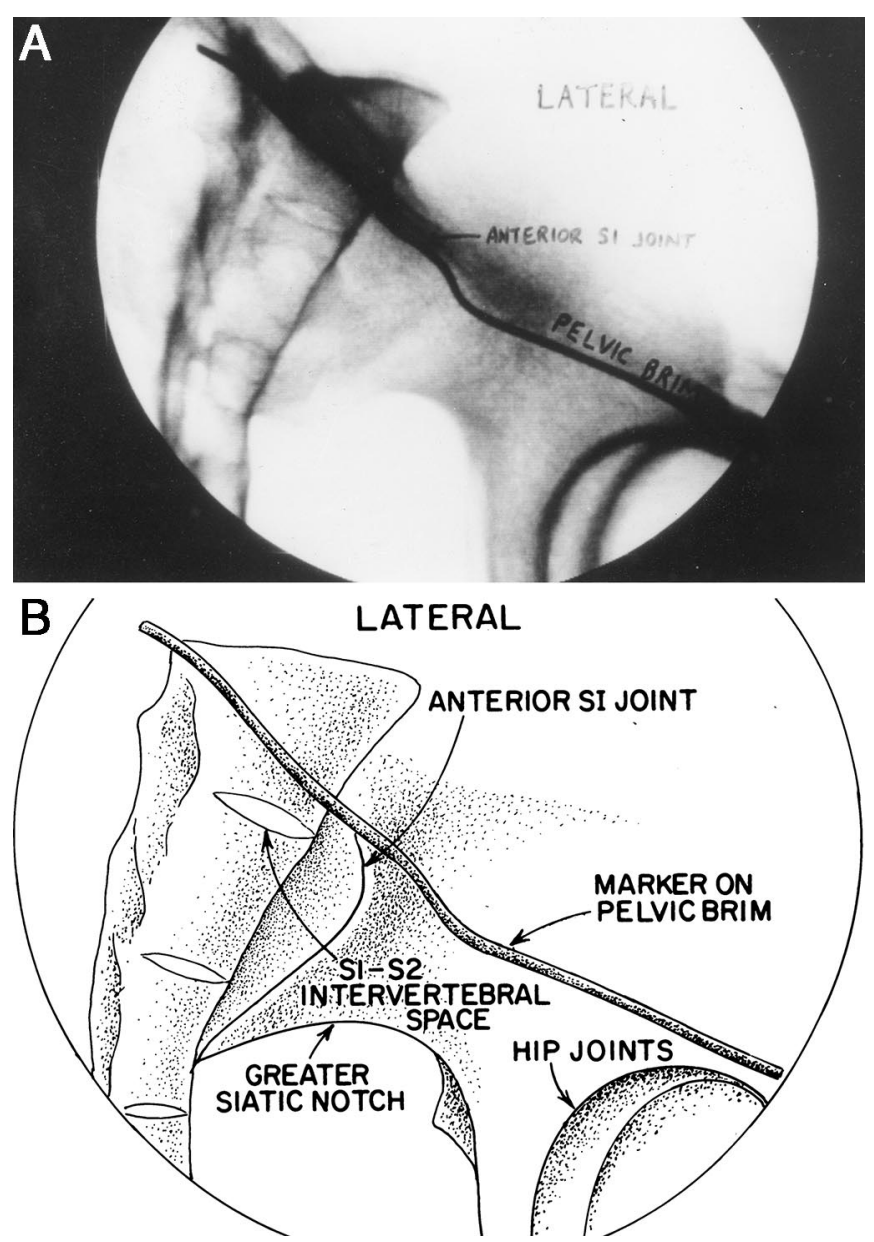

Fig. 10. (A) Alar slope and pelvic brim on lateral view: because of the dense bony sacral structures, the landmarks are poorly delineated and differentiation between left and right sides is difficult. (B) Schematic illustration of the location of the marker placed on the sacroiliac joint and along the pelvic brim.

not identify a distinct line that would consistently identify the true radiographic projection of the alar cortical boundary. The anatomic representation of the dense fluoroscopic line on the lateral view (previously referred to as alar slope or iliac cortical density) corresponded to the general cortical area around the superior aspects of the sacroiliac joint. Anteriorly, the fluoroscopic image of this landmark became confluent with that of the pelvic brim (Fig. 10A, B).

\section{DISCUSSION}

The osseous volume used for iliosacral screw placement is bounded by four surfaces: the posterior S1 body, the superior S1 ala, the anterior S1 ala, and the roof of the $\mathrm{S} 1$ foramen. Although the S1 body is also important, the fact that its prominence and angular orientation differs from that of the smaller corridor of the foraminal region of the sacrum makes it unreliable as a sole reference source. The "bottleneck", so to speak, occurs in the foraminal region of sacrum and has been previously described. ${ }^{15-18}$
If fixation devices exit this volume, structures such as the superior neurovascular bundle (anterior), the L5 nerve root (superior), the S1 nerve root (inferior), and other sacral nerve roots (posterior) are at risk for injury. Although uncommon, the risk of such injuries has been reported. ${ }^{5,13-15,24}$ The conventional method of iliosacral screw placement utilizes the lateral, anterior-posterior, and inlet/outlet views of the pelvis; yet, the scientific basis for the suggested angular orientations of the fluoroscopic beam used for inlet and outlet views has not been established. ${ }^{8,10}$ From our review of the literature, we noted that the only radiographic landmarks advocated for determining the inlet view include the cortices of the anterior and posterior sacral bodies. ${ }^{15}$ Likewise, the sacral foramen may be used as landmarks in the outlet view, but we could not identify any succinct descriptions of how to correctly identify the foramen and what to do in the case of poor visualization.

The 40-degree inlet and outlet views of the pelvis originated from the premise that two plain radiographs oriented approximately 90 degrees to each other provide the most information. The use of 40-degree inlet and outlet views during iliosacral screw placement, however, lacks anatomic support. We have found that the landmarks most pertinent to safe iliosacral screw position are best viewed using inlet and outlet angles that visualize the portion of the bone important to see rather than just moving to a set angle (e.g., 40 degrees). We recognize that our situation is an ideal condition, and in clinical practice, fractures and other anatomic structures may further complicate visualization of this area. We feel that the important fluoroscopic landmarks that require reproducible visualization are those that correspond to the osseous volume of bone used for iliosacral screw placement. The surgeon must be able to reliably visualize these landmarks in each pelvis, independent of the sagittal orientation of the pelvis.

On the inlet view, the angles at which the fluoroscopic beam becomes tangent to the anterior and posterior S1 body (27 and 53 degrees, respectively) both differ from the 40 degrees described in the literature. Mostafavi et al. suggests using the superposition of S2 and S3 bodies, but we could not identify an anatomic basis for their proposal. ${ }^{25}$ Furthermore, because of the tapered and curved shape of the sacrum, we do not think that S2 and S3 structures accurately delineate the boundaries of volume of bone around S1 that is utilized for screw placement. Based on the structures at risk from anterior malposition of the screw, we think that the inlet view should primarily help identify the anterior S1 alar surface (and secondarily, the anterior S1 body). The anterior S1 alar plane was best approximated by the confluence of the S1 and S2 foraminal ridges on the inlet view and averaged 49 degrees in our specimens. We recommend beginning with an anteriorposterior view, where there is easy visualization of the S1 and $\mathrm{S} 2$ ridges. Then by incrementally moving into an inlet direction, our recommendation for the inlet view that best approx- 
imates the anterior S1 alar cortical boundary is when the S1 and S2 foraminal densities super impose. Also, our angular orientations were reported in vitro using a pelvis of fixed orientation, and we recognize that there are in vivo variations in the actual pelvic tilt. On the outlet view, the critical landmarks are the $\mathrm{S} 1$ foramen, the superior ala, and the superior endplate of the S1 vertebral body. The S1 foraminal axis averaged 59 degrees outlet to the AP direction. This is difficult to delineate in vivo because of the large outlet angle required, and because the foraminal axis does not lie exclusively in the sagittal plane and courses obliquely from anterior/lateral/inferior to posterior/medial/superior. The superior ala is visualized at an average of 80 degrees outlet (orientation in the sagittal plane), which, for reasons mentioned previously, is not possible to obtain with the fluoroscope in the clinical setting. Also because of the large variances in the frontal-plane orientation of the superior ala as well as in the variable alar morphology, there may be an increased risk that an iliosacral screw will traverse outside the bony confines of the ala and potentially injure the adjacent L5 nerve root. ${ }^{5,15}$ Nonetheless, because of the difficulties in obtaining an end-on view of the S1 foramen, or an 80-degree outlet view for the superior ala, we propose an alternative method to delineate the structures at risk.

We think that the superior portion of $\mathrm{S} 1$ foramen is the easiest structure to identify in the outlet view and can be used as a reference for screw placement. The superior opening of the $\mathrm{S} 1$ foramen approximates the inferior boundary of the volume of bone used for screw placement. This view is best obtained by starting with an AP view and identifying the dense line representing the $\mathrm{S} 1$ foramen. With incremental outlet views, this opening of the foramen can frequently be seen to emerge and become more spherical. Subjectively, we found the best view to be approximately when the symphyseal image approaches (but does not obscure) the inferior S1 foraminal region (S2 foramen). This view was obtained at around 50 degrees outlet in our in vitro model. We caution, however, that just as with the inlet view, variations in pelvic tilt or the lumbosacral junction would make use of such predetermined angles less reliable in vivo, and that using innate landmarks is preferred. If the trajectory of the fixation device is superior to the $\mathrm{S} 1$ foramen on the outlet view, and within the alar confines on the inlet view, then it is unlikely to violate the anterior or posterior neurovascular structures.

The lateral radiographic view is a key view and has been recommended to determine the starting point of iliosacral screw fixation on the outer ilium, but it is difficult to obtain and interpret. ${ }^{6}$ Because of the potential superimposition of contralateral structures and soft tissues, this region can be very difficult to discern in vivo, especially in overweight patients. We found that the best method to obtain a lateral view is to superimpose radiographic images of the sciatic notches. The sclerotic line coursing anterior-inferiorly has been termed the iliac cortical density or sacral alar slope. ${ }^{7}$ It has been recommended that the screw insertion point begin inferior and posterior to this radiographic density. Previously thought by some to represent the area between the sacroiliac joint and lateral S1 body, we found that this line is not a specific and narrow area, nor a true representation of the iliac or sacral alar cortical boundaries, especially if the surface is irregular in shape. Because radiographic projections are based on the confluence and densities of bone, a convex or concave surface would not image as a dense radiographic line. Likewise, the complex orientation of the sacral ala does not have a surface that is parallel to a laterally directed radiographic beam. Even small adjustments of the beam will only identify small regions of tangential bone and would not be enough to clearly outline the entirety of the sacral ala. Thus, we feel that the fluoroscopic line, called the iliac cortical density or alar slope, represents the general area around the sacroiliac joint and is represented by the confluence of cortical and cancellous bone around the sacroiliac joint. Although not precise, this is the best approximation of the alar cortex on the lateral view.

By beginning an entry point inferior and posterior to this line on the outer ilium, we think the risk of superior/ anterior cortical penetration (and injury to the L5 nerve root) is minimized if the screw is directed properly. It is possible that in a very concave shaped S1 ala, the L5 nerve root would also lie inferior and posterior to this landmark, and would thus be at risk for injury by allowing a screw to enter the ilium, exit the ala medial to the sacroiliac joint (despite appearing to be appropriately placed relative to the alar slope), skirt along the surface of the $\mathrm{S} 1$ ala (where the L5 nerve roots lies), and then re-enter the S1 body. At any time, however, the advocated fluoroscopic views would not give any indication of this aberrance. We would therefore caution that iliosacral screw placement in patients with dysplastic sacra might be dangerous when using fluoroscopic guidance alone. Other techniques such as computed tomographic guidance or evoked potentials may be useful in such situations. ${ }^{2,14,20,24}$ Furthermore, the use of proprioceptive feedback from passing of the drill can help confirm that one is intraosseous. Excessive resistance may indicate a cortical boundary (i.e., foraminal wall or alar cortex) and lack of resistance may indicate an extraosseous position of the drill tip.

The present study was done in vitro and with relatively few specimens, and although there was little variability among the specimens, the wide availability of human anatomic specimens is limited and results in limitations inherent to such studies. The presence of soft-tissue injury and variations of body habitus would affect the ability to visualize the structures reported. Most importantly, the presence of fracture and displacement would significantly affect fluoroscopic visualization. It is because of these problems that we undertook this study in an effort to provide a method of outlining the necessary pelvic structures to aid in the safe placement of iliosacral screws. We did not try to characterize all pelvic structures, as our intent was to only describe the landmarks of 
the bony volume used for iliosacral screw placement. Nonetheless, this study does identify innate relationships that may be useful in clinical practice.

\section{CONCLUSIONS}

Our findings indicate that the standard fluoroscopic views-such as 40 degrees inlet and 40 degrees outlet-do not consistently provide accurate views of the osseous structures required for safe iliosacral screw placement. It is important to note that the value of standard AP views for imaging critical structures is of limited value (especially in viewing S1 foramen). We found that the best inlet view is obtained by incrementally moving from an AP view in the inlet direction, until the radiodense images of the S1 and S2 foraminal ridges (anterior border of the $\mathrm{S} 1$ alar root) are superimposed. The best outlet view is obtained by optimizing an image of the superior ridge of the $\mathrm{S} 1$ foramen. By moving from an AP view in the outlet direction, the radiodense image of the $\mathrm{S} 1$ foramen is followed. As the image of the symphysis approaches this landmark, an image of the $\mathrm{S} 1$ foramen can usually be seen. Most importantly, the lateral view helps identify the superior and anterior sacroiliac joint area (previously referred to as the iliac cortical density or alar slope). The entry point for the screw on the lateral ilium should be posterior to this line, and proprioceptive feedback that helps confirm intraosseous trajectory should be utilized. Although we have been using this method clinically for some time, and we think that the method is easily obtained, reproducible, and anatomically based, the application of such information must be cautiously used in the clinical setting and should be weighed against the surgeon's abilities and experience.

\section{APPENDIX}

Measurements of complex and obliquely oriented structures were made by referencing the $\mathrm{S} 1$ superior endplate as the transverse plane of the sacrum. Planes orthogonal to this plane defined the frontal and sagittal plane of the sacrum. Obviously the sagittal planes for both the sacrum and the pelvis are coplanar. Two structures (S1 ala and S1 foramen) had complex oblique components to their orientation. For the transverse components (anterior S1 ala and S1 foramen), their orientation was measured from a midline reference that was applicable in all sagittal rotations. The transverse measurements were made from fluoroscopic images taken when the structures tangential orientation was obtained. For example, the transverse orientation of the anterior parts of the S1 ala was measured when the pelvis was rotated so that the markers on the anterior ala indicated tangency. In this image, the angle of the ala from the sagittal plane was recorded (Fig. 2A, B). The orientations of the foramen were recorded when the fluoroscopic beam was orthogonal to the axis of the foramen. The angle from the sagittal plane in this view determined the transverse orientation of the foramen. The caudad orientation of the foramen was established by rotating the specimens in the outlet direction until the axis of the tubes was parallel to the transverse axis. Then the specimen was rotated 90 degrees to this in the inlet direction to give a top view of the foramen, and this divergence of the axis of the tubes from the midline established the medial to lateral divergence of the foramen (Fig. 4A, B).

For the frontal component of the superior S1 ala, the superior S1 endplate was used as a reference. As done for the anterior ala, we first obtained a true outlet view (as we described) that identified the tangent to the superior S1 alar surface in the sagittal plane. From here, the frontal component is measured relative to the superior S1 endplate. The planar markers on the superior alar surface, now superimposed, project a line that is sloped medial to lateral and used to define the frontal orientation of the superior alar surface. Recognizing that this surface may be slightly concave or convex, a line connecting the sacral-alar junction to the lateral extent of the markers was used to approximate this surface. As mentioned before, this measurement is in the plane thought of as the "frontal" plane of the sacrum. Although not the "anatomic" frontal plane, the measurements are easier to understand in relation to this sacral reference system as opposed to the pelvic system (Fig. 6). Figure 8 is a schematic representation of the measurements described in Figures 2 through 4 . Note that Figure 2 represents an inlet view and Figure 3 represents an outlet view.

\section{REFERENCES}

1. Matta J, Saucedo T. Internal fixation of pelvic ring fractures. Clin Orthop. 1989;242:83-97.

2. Nelson DW, Duwelius PJ. CT guided fixation of sacral fractures and sacroiliac joint disruptions. Radiology. 1991;180:527-532.

3. Routt ML, Kregor PJ, Simonian PT, Mayo KA. Early results of percutaneous iliosacral screws placed with the patients in the supine position. J Orthop Trauma. 1995;3:207-214.

4. Routt ML, Simonian PT. Closed reduction and percutaneous skeletal fixation of sacral fractures. Clin Orthop. 1996;8:121-128.

5. Routt ML, Simonian PT. Iliosacral screw fixation: early complications of the percutaneous technique. J Orthop Trauma. 1997;11:584-589.

6. Routt ML, Jones CB, Harding SP, Mirza SK. Percutaneous stabilization of U-shaped sacral fractures using iliosacral screws: technique and early results. J Orthop Trauma. 2001;15:238-246.

7. Routt ML, Simonian PT, Agnew SG, Mann FA. Radiographic recognition of the sacral alar slope for optimal placement of iliosacral screws: a cadaveric and clinical study. J Orthop Trauma. 1996;10:171-177.

8. Baque P, Trojani C, Delotte J, et al. Anatomical consequences of "open-book" pelvic ring disruption. A cadaver experimental study. Surg Radiol Anat. 2005;27:487-490.

9. Resnik CS, Stackhouse DJ, Shanmuganathan K, Young JR. Diagnosis of pelvic fractures in patients with acute pelvic trauma: efficacy of plain radiographs. Am J Roentg. 1992;158:109-112.

10. Tile M. Fractures of the Pelvis and Acetabulum. 2nd Ed. Philadelphia: Lippincott Williams and Wilkins; 1995.

11. $\mathrm{Xu} \mathrm{R}$, Ebraheim NB, Douglas K, Yeasting RA. The projection of the lateral sacral mass on the outer table of the posterior ilium. Spine. 1996;21:790-795.

12. Xu R, Ebraheim NB, Robke JK, Yeasting RA. Radiologic evaluation of iliosacral screw placement. Spine. 1996;21:582-588. 
13. Ebraheim NA, Haman SP, Xu R, et al. The lumbosacral nerves in relation to dorsal s1 screw placement and their locations on plain radiographs. Orthop. 2000;23:245-247.

14. Moed BR, Ahmada BK, Craig JG, et al. Intraoperative monitoring with stimulus evoked electromyography during placement of iliosacral screws: an initial clinical study. J Bone and Joint Surg Am. 1998;80:537-546.

15. Templeman D, Schmidt A, Freese J, Weisman I. Proximity of iliosacral screws to neurovascular structures after internal fixation. Clin Orthop. 1996;329:194-198.

16. Amongero ME, Wilber JH. Upper sacral morphology and its relation to sacroiliac screw fixation. Orthopaedic Trans. 1995;19:435.

17. Ebraheim NA, Rongming X, Biyani A, Nadaua MC. Morphologic consideration of the first sacral pedicle for iliosacral screw placement. Spine. 1997;22:841-846.

18. Carlson DA, Scheid DK, Maar DC, et al. Safe placement of s1 and s2 iliosacral screw: The "vestibule" concept. J Orthop Trauma. 2000; $14: 4$.

19. Day CS, Prayson MJ, Shuler TE, et al. Transacral versus modified pelvic landmarks for percutaneous iliosacral screw placement - a computed tomography analysis and cadaveric study. Am J Orthop. 2000;9:16-21.

20. Tonetti J, Carrat L, Lavellee S, et al. Percutaneous iliosacral screw placement using image guided techniques. Clin Orthop. 1998; 354:103-110.

21. Noojin FK, Malkani AL, Haikal L, et al. Cross-sectional geometry of the sacral ala for safe insertion of iliosacral lag screws: a computed tomography model. J Orthop Trauma. 2000;14:31-35.

22. LeTournel E, Judet R. Fractures of the Acetabulum. New York: Springer-Verlag; 1981.

23. Mcollum DE, Gray WJ. Dislocation after total hip arthroplasty: causes and prevention. Clin Orthop. 1990;261:159-170.

24. Webb LX, de Araujo W, Donofrio P, et al. Electromyography monitoring for percutaneous placement of iliosacral screws. J Orthop Trauma. 2000;14:4.

25. Mostafavi H, Tornetta P. Radiographic evaluation of the pelvis. Clin Orthop. 1996;329:6-14.

\section{EDITORIAL COMMENT}

The three-dimensional anatomy of the pelvis is difficult to visualize with two-dimensional radiography. This study assists pelvic surgeons in placing iliosacral screws by providing a better understanding of what we see fluoroscopically during surgery. The authors used radiodense markers and pointers to correlate anatomic landmarks with radiographic density and lucency in cadaveric pelvises. The fluoroscopic beam was moved to define the orientation of tangential bony surfaces in critical areas.

The authors discovered several important points. First, it is not safe to use predetermined inlet and outlet angles to view the pelvis, as there will be variation in sacral morphology and lumbosacral lordosis between individuals. Second, the anterior border of the sacral ala is not parallel to the anterior border of the $\mathrm{S} 1$ body. Surprisingly, it is nearly parallel to the posterior border of the S1 body. Third, several areas (i.e., the posterior S1 body, the superior sacral ala, and the posterior foraminal region of S1) are difficult to visualize fluoroscopically.

In their discussion, Ziran et al. emphasize that by incrementally moving the fluoroscopic angle, the surgeon can obtain modified inlet and outlet views that will best display the bony surfaces that must not be violated by guidewires or screws. Although these concepts may already be understood by experienced pelvic surgeons, the data and documentation provided serve to strengthen the foundation of available knowledge. Thus, this study is an important reference for all pelvic surgeons.

John Gorczyca, MD

University of Rochester School of Medicine

Rochester, New York 